CERN-TH.7448/94

BARI-TH/182-94

\title{
Technicolour and Precision Electroweak Data Revisited
}

\author{
John Ellis ${ }^{a}$, G.L. Fogli ${ }^{b}$ and E. Lisi ${ }^{a, b}$ \\ a Theory Division, CERN, CH-1211, Geneva, Switzerland \\ ${ }^{b}$ Dipartimento di Fisica and Sezione INFN di Bari, Bari, Italy
}

\begin{abstract}
We examine the present status of technicolour (TC) models in the light of recent improvements both in theory and experiment. First we present an updated modelindependent fit of high-energy precision electroweak data, and emphasize their compatibility with the Standard Model. We then compare the model-independent fit with a one-generation TC model with $N_{T C}=2$, degenerate techniquarks and either a Dirac or a Majorana technineutrino, not necessarily degenerate with the technielectron. The results are charted in a way which clearly shows how data disfavour simple TC models, unless judicious combinations of additional effects (induced by the dynamics and/or the spectrum) are allowed. Techniquark mass splitting is shown to be fatal to the Dirac case. Even allowing for theoretical uncertainties, a pattern of stringent constraints on TC models is seen to emerge.
\end{abstract}

CERN-TH.7448/94

BARI-TH/182-94

October 1994 
One of the key issues in electroweak physics now is the mechanism of symmetry breaking and mass generation. Theoretically, one can distinguish two broad lines of thought: one is that there is an elementary Higgs boson, accompanied presumably by supersymmetric particles, and the other is that electroweak symmetry breaking is dynamical in nature, due to the condensation in the vacuum of some stronglyinteracting fermions, in which case the Higgs boson is replaced by a composite state (see Ref. [1] for a recent review of this approach). The condensing fermions might either be heavy top quarks with strong Yukawa interactions, as in $\bar{t} t$ condensate models [2], or new fermions with extra strong gauge interactions, commonly known as technicolour models (TC) [3].

Theoretical calculations in elementary Higgs models are in principle less ambiguous than in models of dynamical symmetry breaking, precisely because the latter are strongly-interacting and hence trickier to calculate reliably. Moreover, technicolour models require extensions $[4,5]$ if they are to explain quark and lepton masses, and there is no single preferred scenario for this such an extension, which must moreover be crafted in such a way as to respect the important experimental constraints on flavour-changing neutral interactions [5, 6]. On the other hand, the Higgs sector in the minimal Standard Model (SM) contains just one parameter, which can be identified with the Higgs mass, and reliable calculations are feasible unless this parameter is large. Calculations in the Minimal Supersymmetric extension of the Standard Model (MSSM) depend on more parameters, but can again be made reliably, because the parameters may always be treated in perturbation theory.

Until the advent of precision electroweak data from LEP and elsewhere, there was no real experimental clue favouring the elementary Higgs scenario over composite models, or vice versa. However, it has become apparent that these data are consistent with weakly-coupled elementary Higgs models such as the SM [7, 8, 9] or the MSSM [8, 10], whilst disfavouring strongly-coupled dynamical models of electroweak symmetry breaking [11]. The extent of this disfavour is difficult to quantify, because of the above-mentioned uncertainties in non-perturbative calculations and variety of models. Moreover, the evolutionary pressure due to the electroweak data 
has caused technicolour modellers to refine and adapt their models and calculations (see e.g. $[12,13]$ ), and there have been improvements in the phenomenological parametrization of possible effects from beyond the SM. It therefore appears to us opportune to re-examine now the extent to which technicolour models are compatible with the precision electroweak data.

The general approach to confronting technicolour models with precision electroweak data that we follow is based on the so-called "model-independent" parametrization of vacuum polarizations and vertex corrections. This is based on the observation that, after fixing the electroweak renormalization conditions, removing purely QED+QCD corrections and making a low-momentum series expansion in the vacuum polarization (oblique) diagrams, there are three independent oblique parameters [14], named either $S, T, U[15]$ or $\epsilon_{1,2,3}$ [16], to be constrained by the precision electroweak data at a scale $Q=M_{Z}$. This observation has been extended to include a non-oblique parameter describing $Z \rightarrow \bar{b} b$ decay, called $\epsilon_{b}$ [17], which we shall also include in our analysis. This framework has recently been further expanded by the introduction of additional parameters $V, W, X[18,19]$ to describe the lowest non-trivial order momentum dependence in the oblique diagrams. However, these are currently of limited usefulness, as we shall discuss later.

It is well known that the magnitude of the technicolour radiative corrections increases with the number of technicolours $N_{T C}$ and the number of techniflavours $N_{T F}$ (see [15] and references therein). We take the point of view that the minimal possibility is a one-technigeneration model with $N_{T C}=2$, and use this as our reference technicolour model. It contains a colour triplet of techniquarks $(U, D)$ and a doublet of technileptons $(N, E)$. Naïvely, one could expect that $M_{U}-M_{D} \simeq m_{t}$, but this would produce a very large shift in the variable $\epsilon_{1}$ (or $T$ ) which is sensitive to electroweak isospin splitting, and is not required theoretically. In this paper we will generally assume the most favourable situation $M_{U}=M_{D}$, whilst commenting on the implications of a splitting with $M_{U}>M_{D}$. There are two popular ways of varying the technilepton sector: the technineutrino $N$ may be either Dirac [15] or Majorana [20], and there may be isospin splitting $M_{N}<M_{E}$. We study both possibilities in this paper, and discuss what relationship between $M_{N}$ and $M_{E}$ is least disfavoured by experiment. 
We find that all the above variations on the basic one-technigeneration model are experimentally disfavoured, at least if naïve calculations of the magnitudes of technicolour radiative corrections are used. However, some suggestions have been made to improve this situation, involving ad hoc effects from the bosonic spectrum and/or from the dynamics of the theory, as in the walking [21] or strong [22] extensions of technicolour. As we will see later, a judicious combination of these effects would be needed to bring the class of technicolour models that we study into agreement with the precision electroweak data.

Before discussing the results in detail, we first review relevant elements of the formalism that we use.

The starting point for the type of "model-independent" approach that we follow here is a Born approximation in which all the one-loop QED effects are absorbed, principally the running of $\alpha$ between the Thompson limit and a renormalization scale $Q=M_{Z}: \bar{\alpha}=\alpha\left(M_{Z}\right) \simeq 1 / 128.87$. The corresponding value of the electroweak mixing angle to be used in estimating the one-loop radiative corrections is $\vartheta_{0}$ :

$$
\sin ^{2} \vartheta_{0} \cos ^{2} \vartheta_{0} \equiv s_{0}^{2} c_{0}^{2}=\frac{\pi \bar{\alpha}}{\sqrt{2} G_{F} M_{Z}^{2}}
$$

Moreover, QCD corrections up to $\mathcal{O}\left(\alpha_{s}^{3}\right)$ are absorbed in the hadronic decay widths of the $Z$ boson. The genuine electroweak radiative corrections correspond to deviations from this Born approximation, which are parametrized in the "model-independent" approach by $\epsilon_{b}$ and $\epsilon_{1,2,3}$ or $(S, T, U)$ in the one-loop approximation.

In the minimal SM the radiative corrections are specified by the assumed values of $m_{t}$ and $M_{H}$. Within a specific theoretical framework like the SM or the MSSM it is possible to go beyond the one-loop level, and include a resummation of the dominant higher-loop effects, which means that the model-independent approach is not the most accurate way to analyze the parameters of the SM or the MSSM. Moreover, it is not suited to incorporate low energy data $\left(Q \ll M_{Z}\right)$. However, the $\epsilon_{i}$-parametrization is perfectly adequate when maximum precision and information are not required, as in the analysis of TC models. 
Reference values of $m_{t}$ and $M_{H}$ must be assumed in order to separate TC contributions $\delta \epsilon_{i}$ from the SM parts of the one-loop radiative corrections to the Born approximation: $\epsilon_{i}=\epsilon_{i}^{S M}\left(m_{t}, M_{H}\right)+\delta \epsilon_{i}$. The relations between the definitions of $(S, T, U)$ and the $\delta \epsilon_{i}$ are then given by:

$$
\left\{\begin{array}{l}
\delta \epsilon_{1}=\epsilon_{1}-\epsilon_{1}^{S M}=\bar{\alpha} T \\
\delta \epsilon_{2}=\epsilon_{2}-\epsilon_{2}^{S M}=-\bar{\alpha} U / 4 s_{0}^{2} \\
\delta \epsilon_{3}=\epsilon_{3}-\epsilon_{3}^{S M}=\bar{\alpha} S / 4 s_{0}^{2}
\end{array} .\right.
$$

Data from CDF [23] and a more accurate SM analysis [8] of the precision electroweak data indicate that $m_{t} \simeq 170 \mathrm{GeV}$, so we take this as our reference value of $m_{t}$. The SM requires $63 \mathrm{GeV} \leq M_{H} \lesssim 1000 \mathrm{GeV}$, with the precise $\mathrm{SM}$ analysis favouring a value $M_{H} \lesssim M_{Z}$, so we take $M_{H}=M_{Z}$ as our reference value.

As already mentioned above, an extension of the above parametrization to include the lowest non-trivial orders in the momentum dependences of the relevant vacuum polarizations has been proposed, with the motivation of treating extensions of the SM that contain additional low-mass particles. Three new parameters $V, W, X$ are introduced $[18,19]$, involving shifts in the vector boson polarizations and their derivatives at $Q=0, M_{Z}$. The parameter $W$ appears in the decay width of the $W$ boson, but not in the precision electroweak observables studied here. Concerning $X$, explicit calculations in ETC models $[19,24]$ find that this parameter is very small in all scenarios, so it can also be neglected. The parameter $V$ may become significant for small technifermion masses [24] $M_{N, E, U, D} \lesssim M_{Z}$. However, we regard this possibility as unlikely, at least to the extent that these masses can be rescaled from constituent quark masses in QCD. We note, moreover, that even if this extended parametrization allows the incorporation of low-energy data $(Q \simeq 0)$ in addition to the more precise high-energy data discussed below $\left(Q \simeq M_{Z}\right)$, a fit including up to seven free parameters $\left(\epsilon_{1}, \epsilon_{2}, \epsilon_{3}, \epsilon_{b}, V, W, X\right)$ would be only poorly constrained. We therefore restrict our attention to the basic parameters $\epsilon_{1}, \epsilon_{2}, \epsilon_{3}$ (or $S, T, U$ ) and $\epsilon_{b}$, assuming that $V, X \simeq 0$. 
The input data that we use in our fits have been presented at the 1994 Glasgow International Conference on High Energy Physics. They include the combined $\mathrm{CDF}+\mathrm{D} 0+\mathrm{UA} 2$ measurements of $M_{W}$ [25], the LEP determinations of $\Gamma_{Z}$, $\sigma_{h}^{0}, \Gamma_{b} / \Gamma_{h}, \Gamma_{c} / \Gamma_{h}$ and $R_{h}=\Gamma_{h} / \Gamma_{e}[26]$, and the combined LEP+SLD value of $g_{V} / g_{A}$ from peak asymmetries [26, 27]. In addition, we use the matrix of correlations between the LEP data provided by the LEP Electroweak Working Group, and assume $\alpha^{-1}\left(M_{Z}\right)=128.87 \pm 0.12$ [28] and $\alpha_{s}\left(M_{Z}\right)=0.118 \pm 0.007$ [29]. The errors in these quantities are propagated and included with the experimental errors in calculating the value of $\chi^{2}$. Our fit yields

$$
10^{3} \times\left\{\begin{aligned}
\epsilon_{1} & =4.5 \pm 1.9 \\
\epsilon_{2} & =-5.1 \pm 5.2 \\
\epsilon_{3} & =4.8 \pm 1.8 \\
\epsilon_{b} & =2.4 \pm 3.9
\end{aligned}\right.
$$

with $\chi_{\min }^{2}=2.4$ and a correlation matrix

$$
\rho_{\epsilon}=\left(\begin{array}{cccc}
1 & & & \\
0.26 & 1 & & \\
0.86 & 0.15 & 1 & \\
-0.23 & -0.07 & -0.15 & 1
\end{array}\right),
$$

and is displayed graphically in Fig. 1. In attempt to portray the salient aspects of the fit in the four-dimensional space, we display all 6 projections of the $\Delta \chi^{2} \equiv$ $\chi^{2}-\chi_{\min }^{2}=1$ ellipsoid defined by equations (3), (4) on the planes $\left(\epsilon_{i}, \epsilon_{j}\right)$. These projections are shown as solid-line ellipses in the different planes, linked by dotted lines that connect the appearances of the same variable $\epsilon_{i}$.

Also shown in each planar projection is a star at the position of the Born approximation. This clearly lies far outside the $\Delta \chi^{2}=1$ ellipsoid, and corresponds in fact to $\Delta \chi^{2}=10.6$. We conclude that pure electroweak radiative corrections have indeed been seen at the level of $\sqrt{\Delta \chi^{2}}=3.3$ standard deviations ${ }^{1}$, although both $\epsilon_{b}$

\footnotetext{
${ }^{1}$ Here and in the following we use the term "standard deviation" $(\sigma)$ in a broad sense, as in Ref. [30]. It is understood that in a multiparametric analysis like ours, the confidence level associated to an iso- $\sigma$ contour depends on the number of free parameters involved [31].
} 
and (marginally) $\epsilon_{2}$ are compatible with zero. Also shown in each planar projection is the range of values of the $\epsilon_{i}$ calculated in the $\mathrm{SM}$ with $140 \mathrm{GeV} \leq m_{t} \leq 200 \mathrm{GeV}$ and $60 \mathrm{GeV} \leq M_{H} \leq 1000 \mathrm{GeV}$. We see that this overlaps well with the fit ellipsoid, except in the projection on the $\epsilon_{b}$ axis. This reflects the well-known fact that the data do not correspond well to the value of $\Gamma(Z \rightarrow \bar{b} b)$ expected in the SM for $m_{t}$ in the above range. Within the SM, we find $\Delta \chi^{2}=6.8$ at the reference point, the value of $\Delta \chi^{2}$ being slightly improved for somewhat lower values of $\left(m_{t}, M_{H}\right)$. We conclude that there is no significant disagreement with the SM. However, we re-emphasize that the best way to confront the SM with the precision electroweak data is to use exactly all the available theoretical calculations, including those of higher loops, and also to use all the world's available data, including those from lower energies ${ }^{2}$. When this is done, one finds an excellent fit to both low- and high-energy data and confidence regions in the $\left(m_{t}, M_{H}\right)$ plane as in Ref. [8].

Before discussing the implications of our fits for various technicolour models, we first collect the theoretical formulae that we use for $(S, T, U)$ and $\delta \epsilon_{b}=\epsilon_{b}-\epsilon_{b}^{S M}$. As already mentioned, we discuss two scenarios, which have identical techniquark sectors but either a Dirac or a Majorana technineutrino. In the case of Dirac $(D)$ technileptons $(N, E)$, one-loop perturbative calculations [15] yield

$$
\begin{aligned}
S_{D} & =\frac{N_{T C}}{6 \pi}(1-Y \ln r), \\
T_{D} & =\frac{N_{T C}}{16 \pi s_{0}^{2} c_{0}^{2}} \frac{M_{E}^{2}}{M_{Z}^{2}}\left[1+r-2 \frac{r}{r-1} \ln r\right], \\
U_{D} & =\frac{N_{T C}}{6 \pi}\left[-\frac{5 r^{2}-22 r+5}{3(r-1)^{2}}+\frac{r^{3}-3 r^{2}-3 r+1}{(r-1)^{3}} \ln r\right] .
\end{aligned}
$$

where $r \equiv M_{N}^{2} / M_{E}^{2}$ and $Y$ is the weak hypercharge of the $(N, E)$ doublet. Similar formulae apply to the techniquark doublet $(U, D)$, with an extra factor of $N_{C}$ and

\footnotetext{
${ }^{2}$ If the $Q$ dependence of the vacuum polarization derivatives in the range $0 \lesssim Q \lesssim M_{Z}$ is neglected, then low-energy data can be $\epsilon_{i}$-parametrized. In this case the central fit value of $\epsilon_{3}$ would be slightly lower.
} 
the appropriate value of $Y$. In the limit $\Delta M=M_{E}-M_{N} \ll M_{E}$, these formulae reduce to the expressions

$$
\begin{aligned}
& S_{D} \simeq \frac{N_{T C}}{6 \pi}, \\
& T_{D} \simeq \frac{N_{T C}}{12 \pi s_{0}^{2} c_{0}^{2}} \frac{\Delta M^{2}}{M_{Z}^{2}}, \\
& U_{D} \simeq \frac{2 N_{T C}}{15 \pi} \frac{\Delta M^{2}}{M_{E}^{2}} .
\end{aligned}
$$

The expression for $S$ is not expected to be applicable directly to the data, as it is subject to significant non-perturbative corrections. Spectral function techniques [15, 32] suggest that each (degenerate) doublet of Dirac technifermion contribute $\Delta S_{D} \simeq$ $0.1 N_{T C}$, that is almost twice the perturbative estimate (8). Formally different results are obtained with chiral lagrangian techniques [33], although in the one-generation model considered here the value of $S$ is numerically at least twice as large as in (8). An independent calculation [34] tends to confirm the estimate $0.1 N_{T C}$ per doublet, at least in models with dynamics rescaled from QCD. Therefore we assume, for the values of $S$ associated with $(U, D)$ and $(N, E)$ in the degenerate limit $M_{E}=M_{N}$

$$
S_{\text {deg }}=0.1\left(N_{C}+1\right) N_{T C}=0.8,
$$

whereas $T$ and $U$ vanish in this limit. For generic values of $M_{E}, M_{N}$ we take

$$
S=S_{d e g}+\left(S_{D}-\frac{N_{T C}}{6 \pi}\right)
$$

thus matching Eq. (11) for $M_{E}=M_{N}$, and use the perturbative estimates of $T$ and $U\left(T=T_{D}\right.$ and $\left.U=U_{D}\right)$.

The above formulae are modified if the technineutrino $N$ is a Majorana $(M)$ particle [20], as would happen if there were a massive singlet right-handed technineutrino $N_{R}$ that mixed with $N$, via a typical $2 \times 2$ see-saw mass matrix with off-diagonal Dirac mixing term $M_{D}$. If one assumes that $M_{D}=M_{E}$, one finds the following modifications of the equations (5)-(7) above: 


$$
\begin{aligned}
& S_{M}= \frac{N_{T C}}{6 \pi}\left\{-\frac{r}{(1+r)^{2}}\left[\frac{8}{3}+\frac{3 r-4 r^{2}+3 r^{3}}{\left(1-r^{2}\right)^{2}}+2 \frac{r^{6}-3 r^{4}+6 r^{3}-3 r^{2}+1}{\left(1-r^{2}\right)^{3}} \ln r\right]\right. \\
&\left.+\frac{1-r}{1+r} \ln r+\frac{3}{2}\right\}, \\
& T_{M}= \frac{N_{T C}}{16 \pi s_{0}^{2} c_{0}^{2}} \frac{M_{E}^{2}}{M_{Z}^{2}}\left\{2-\frac{4 r}{r^{2}-1} \ln r+\frac{4 r}{(r+1)^{2}}\left[1-\frac{r^{2}+1}{4 r}-\frac{r^{2}-r+1}{r^{2}-1} \ln r\right]\right\}, \\
& U_{M}=\frac{N_{T C}}{6 \pi}\left\{\frac{r}{(r+1)^{2}}\left[\frac{8}{3}+\frac{3 r^{3}-4 r^{2}+3 r}{\left(r^{2}-1\right)^{2}}-2 \frac{r^{6}-3 r^{4}+6 r^{3}-3 r^{2}+1}{\left(r^{2}-1\right)^{3}} \ln r\right]\right. \\
&\left.+\frac{r^{3}-3 r^{2}-3 r+1}{(r-1)^{3}} \ln r-\frac{13}{6}+\frac{4 r}{(r-1)^{2}}\right\} .
\end{aligned}
$$

In the limit of small mass splitting $M_{E}-M_{N} \ll M_{E}$, these formulae become:

$$
\begin{aligned}
& S_{M} \simeq \frac{N_{T C}}{6 \pi}, \\
& T_{M} \simeq-\frac{N_{T C}}{12 \pi s_{0}^{2} c_{0}^{2}} \frac{\Delta M^{2}}{M_{Z}^{2}}, \\
& U_{M} \simeq-\frac{2 N_{T C}}{15 \pi} \frac{\Delta M^{2}}{M_{E}^{2}} .
\end{aligned}
$$

Note that whilst the formula (16) for $S$ is identical with the Dirac case (8), the formulae for $T$ and $U$ change sign. This point is important, since it improves, in particular, the prospects of agreement with the experimental value of $\epsilon_{3}$, as we shall see later. The above formulae are again subject to non-perturbative corrections. In the case of $S$, one estimate [35] indicates that about $0.04 N_{T C}$ should be added to the perturbative technilepton contribution $S_{M}$, as well as the previous $0.1 N_{C} N_{T C}=0.6$ from the Dirac techniquark sector. Thus we adopt the following estimate for the total value of $S$ in the Majorana case:

$$
S=\left(0.04+0.1 N_{C}\right) N_{T C}+S_{M} .
$$

In the case of $T_{M}$, a non-perturbative estimate [36] is apparently similar to the perturbative one above, which we use in our analysis $\left(T=T_{M}, U=U_{M}\right)$. 
Concerning the evaluation of the $Z \rightarrow \bar{b} b$ vertex in TC models, one should take both ETC vector boson and pseudo-Nambu-Goldstone boson (PNGB) exchange into account. The main contribution, which comes from exchange of those ETC bosons causing fermion-technifermion transitions, is approximately given by [37]:

$$
10^{3} \times \delta \epsilon_{b} \simeq-24 \xi^{2}\left(m_{t} / 170 \mathrm{GeV}\right)
$$

where $\xi$ is a Clebsch-Gordan coefficient that must be of order unity. In addition, there is a correction of similar form [38], coming from ETC bosons diagonally interacting with techni- and ordinary fermions. It is estimated to change $\xi$ by an amount

$$
\xi^{2} \rightarrow \xi^{2}+\frac{4}{(4 \pi)^{\frac{4}{3}}} \frac{N_{C} N_{T C}}{N_{T C}+1}
$$

if the technicolour group is $S U\left(N_{T C}\right)$ and the ETC group is $S U\left(N_{T C}+1\right)$. Finally, there is a further negative contribution to $\epsilon_{b}$ from charged PNGB exchange [39]. This shift, whose magnitude increases with both $m_{t}$ and $N_{T C}$, ranges from a few per mill (heavy PNGB spectrum) to a few per cent (light PNGB spectrum). In comparing TC models with data, we assume that total TC contribution $\delta \epsilon_{b}$ is given at least by Eq. (20) with $\xi \geq 1 / 2$, i.e. $\delta \epsilon_{b} \lesssim-6 \times 10^{-3}$.

We are now equipped to compare the experimental values of the $\epsilon_{i}$ with the above technicolour predictions. In making this comparison, we shall mention various possible modifications of the technicolour predictions that could reduce their disagreement with experiment. Figure 2 is similar to Figure 1, but with the following changes. The projections of the $\Delta \chi^{2}=1,4$ ellipsoids on the different $\left(\epsilon_{i}, \epsilon_{j}\right)$ planes are both shown, and the stars representing the Born approximation have been moved to corners of the planar projections, so as to display more clearly the quadrants in which lie the technicolour predictions for the Dirac technineutrino case. These are shown as arrays of dots corresponding to models obeying the constraints

$$
100 \mathrm{GeV} \leq M_{E} \leq 600 \mathrm{GeV} \quad, \quad 50 \mathrm{GeV} \leq M_{N} \leq M_{E} \quad, \quad 1 / 2 \leq \xi \quad .
$$

The arrows represent various possible modifications of these predictions that we shall discuss in due course. 
Now we comment on the main results emerging from Fig. 2.

1) First we note that the Dirac technicolour model fits the data considerably worse than the SM. In most of the parameter space (22), $\epsilon_{1}$ is too large, although it does approach the $\Delta \chi^{2}=1$ ellipse if $M_{N} \simeq M_{E}$. The value of $\epsilon_{2}$ is also acceptable in this limit, but we see from the $\left(\epsilon_{1}, \epsilon_{3}\right)$ and $\left(\epsilon_{2}, \epsilon_{3}\right)$ projections that the value of $\epsilon_{3}$ is too high in this limit. There are some Dirac technicolour models with small enough $\epsilon_{3}$, but these have disfavoured values of $\epsilon_{1}$ and/or $\epsilon_{2}$.

2) We also note that the value of $\epsilon_{b}$ is always too negative, indeed considerably worse than in the SM. Just how much worse depends on the model's value of $\xi$ : a model with $\xi=1 / 3$ would be only slightly worse than the SM, whereas a model with $\xi=1$ would not even appear on our restricted planar projections.

3) We now comment on the arrows in Fig. 2: these represent possible modifications of the above predictions in variants of technicolour models, with a particular emphasis on those contributions which appear more reliable and/or motivated (bold arrows). The bold arrow labelled TQ recalls that $\epsilon_{1}$ is expected to increase rapidly with techniquark splitting $M_{U}>M_{D}$ in a variety of TC realizations [15, 40], worsening the consistency with experiments. The bold arrow labelled $\mathrm{B}$ emphasizes that the additional bosonic contributions $[38,39]$ to $\epsilon_{b}$ also worsen the discrepancy with experiment. The thinner arrows labelled $\mathrm{B}$ remind the reader that ad hoc bosonic effects may either increase or decrease the value of $\epsilon_{3}$, for example if technifermions are non-degenerate $[41,42]$ or depending on the masses of the $\rho_{T}$ and $\omega_{T}$ [43], or provide a welcome possible decrease in $\epsilon_{1}$ [41]. The thin arrow labelled NC draws attention to the very welcome (but more exotic) possibility that $\epsilon_{b}$ could be decreased in non-commuting ETC models [44]. It is apparent from Fig. 2 that both this and a decrease in $\epsilon_{3}$ due to bosonic corrections would be needed to bring the Dirac model into agreement with experiment.

Figure 3 presents a parallel analysis of models with a Majorana technineutrino. It is apparent that such models may fare better than the Dirac models in figure 2, though still worse than the SM. The main reason for this is that eqs. (13), (14) allow a simultaneous reduction in $\epsilon_{1}$ and $\epsilon_{3}$. As we discuss later, this even allows scope for $M_{U}>M_{D}$, which is essentially impossible in the Dirac model. Although the 
formal expressions for $\epsilon_{2}$ and $\epsilon_{3}$ differ from those in the Dirac model, they are still strongly correlated. In particular, it is still true that either $\epsilon_{2}$ or $\epsilon_{3}$ may fall within the experimentally acceptable range, but not both simultaneously. As in Fig. 2, the arrows in Fig. 3 represent possible modifications of the standard technicolour calculations used up to now. The bold arrow labelled TQ reminds again the reader of the feature that non-degenerate techniquarks would increase $\epsilon_{1}$. The bold arrows labelled WTC-SETC recall that $\epsilon_{b}$ can be reduced in certain walking [45] and strong extended [46] technicolour models. WTC models may also either increase or decrease the value of $\epsilon_{3}$ [34], and/or decrease $\epsilon_{1}$ [47], but these effect are quite uncertain (thin WTC arrows). The former property could be used to reconcile such a technicolour model with the experimental constraint in the $\left(\epsilon_{2}, \epsilon_{3}\right)$ plane.

We emphasize that the arrows in Figs. 2, 3 all apply to both the Dirac and Majorana technineutrino models: it is only for graphical convenience that they were displayed separately. Of course, the desirability of such a modification depends on the scenario studied.

How significant is the disfavour with which the data view these technicolour models? We attempt an answer in Fig. 4, which displays contours of equal $\sigma \equiv \sqrt{\Delta \chi^{2}}$ in the $\left(M_{E}, M_{N}\right)$ planes for the Dirac and Majorana models. In this figure, (a) and (b) refer to the cases studied above in which $M_{U}=M_{D}$, whereas (c) and (d) consider the possibility that $M_{U}>M_{D}$, in such a way that they contribute ${ }^{3} 0.01$ to $\epsilon_{1}$.

We first consider the two subfigures (a), (b). It can be seen that both the Dirac and Majorana models have $\sigma \gtrsim 4.5$, although the preferred values of $M_{E}, M_{N}$ are rather different. The Dirac model (a) prefers a "moderate" mass splitting $M_{E}-M_{N} \simeq 80 \mathrm{GeV}$, whereas the Majorana model (b) favours $M_{E} \ll M_{N}$. If we now add 0.01 to $\epsilon_{1}$, as a representative effect of $M_{U}>M_{D}$, then the values of $\sigma$ in the Dirac model increase by about 4 units, and $M_{E} \simeq M_{N}$ is required to avoid further positive contributions to $\epsilon_{1}$, as it is evident in (c). Conversely, in the Majorana model $(d)$ it is still possible to fit the increased value of $\epsilon_{1}$ at $\sim 5 \sigma$ level. We have assumed $\xi=1 / 2$ throughout Figure 4: decreasing $\xi$ to $1 / 3$ would reduce $\sigma$ by about one unit, whilst increasing $\xi$ to 1 would increase $\sigma$ by about 4 units. We

\footnotetext{
${ }^{3}$ Note that for $M_{U}-M_{D} \simeq m_{t}$ a simple perturbative estimate would give an unacceptably large contribution $\delta \epsilon_{1} \simeq 0.024$.
} 
have not exhibited the region of the $\left(M_{E}, M_{N}\right)$ plane with inverted mass splitting $M_{E}<M_{N}$ : in this region $\sigma \gtrsim 7$ in the Dirac case, and $\gtrsim 5$ in the Majorana case, with the minimum being reached for unacceptably small values of $M_{E}$.

Let us draw now our conclusions. We have seen in this paper that even the minimal technicolour models, with $N_{T C}=2$ and just one technigeneration, have difficulties obtaining computable radiative corrections $\delta \epsilon_{i}$ that are small enough to accommodate the precision electroweak data and $m_{t}=170 \mathrm{GeV}$, without fine tuning and $a d$ hoc assumptions. Existence proofs have been produced [48], but these exploit the fact that experiment allows relatively large values of $S, T$ and $U$ if additional parameters $V, W$ and $X$ are left free, whereas models generally predict small values of $V$ and $X$. It should also be noted that we have not addressed the additional experimental constraints on FCNC, nor attempted to impose unification of the coupling strengths in TC models [49], which could be a source of additional problems. We finally note that some authors have recently proposed that CDF may be seeing an unexpectedly high $\bar{t} t$ production cross-section [23] as a result of a direct-channel colour-octet bosonic resonance [50]. If true, this should be taken as an extra constraint on TC model-building, but the upper limit on the $\bar{t} t$ cross-section announced by D0 [51] disfavours this interpretation in any case.

The $a d$ hoc assumptions required to reconcile TC models with experiment must be weighed against its natural advantages. This is in contrast with the MSSM, which is consistent with precision electroweak data and grand unification [52] (as well as with $\mathrm{FCNC}$ constraints) in a variety of realizations. It must be noted that also $\bar{t} t$ condensate models, predicting $m_{t} \gtrsim 220 \mathrm{GeV}$ with the most favourable (highest) scale of new physics [2], are now ruled out at more than 3 standard deviation level by the CDF kinematic fit of $m_{t}$, as well as precision electroweak measurements. It is thus evident that present experimental data do not support composite Higgs models and clearly favour elementary Higgses as in the SM and its supersymmetric extension. This gives us more confidence in the possibility of estimating the elementary Higgs boson mass from precision electroweak data [8], while waiting hopefully for its discovery at LEPII or the LHC. 


\section{Acknowledgements}

One of us (E.L.) wishes to thank the CERN Theory Division for their kind hospitality during all stages of this work.

\section{References}

[1] S.F. King, Univ. of Southampton preprint SHEP 93/94-27, submitted to Rep. Prog. Phys., and references therein.

[2] K. Yamawaki, in Proceedings of the Workshop on Effective Field Theories of the Standard Model (Dobogókö, Hungary, 1991), ed. by U.-G. Meißner (World Scientific, Singapore, 1992), p. 307, and references therein.

[3] S. Weinberg, Phys. Rev. D19 (1979) 1277;

L. Susskind, Phys. Rev. D20 (1979) 2619;

E. Fahri and L. Susskind, Phys. Rep. 74 (1981) 2677.

[4] S. Dimopoulos and L. Susskind, Nucl. Phys. B155 (1979) 237.

[5] E. Eichten and K. Lane, Phys. Lett. B90 (1980) 125.

[6] S. Dimopoulos and J. Ellis, Nucl. Phys. B182 (1981) 505.

[7] J. Ellis, G.L. Fogli and E. Lisi, Phys. Lett. B292 (1992) 427; B318 (1993) 148.

[8] J. Ellis, G.L. Fogli and E. Lisi, Phys. Lett. B333 (1994) 118.

[9] G. Montagna, O. Nicrosini, G. Passarino and T. Piccinini, Phys. Lett. B335 (1994) 484 .

[10] J. Ellis, G.L. Fogli and E. Lisi, Phys. Lett. B286 (1992) 85; Nucl. Phys. B393 (1993) 3.

[11] J. Ellis, G.L. Fogli and E. Lisi, Phys. Lett. B285 (1992) 238. 
[12] K. Lane, Boston Univ. preprint BUHEP-94-2, to appear in Proceedings of 1993 TASI Lectures (World Scientific, Singapore).

[13] T. Appelquist and J. Terning, Phys. Rev. D50 (1994) 2116.

[14] B.W. Lynn, M.E. Peskin and R.G. Stuart, in Physics at LEP, ed. by J. Ellis and R. Peccei (CERN Report CERN-86-02, Geneva, 1986).

[15] M.E. Peskin and T. Tacheuchi, Phys. Rev. D46 (1992) 381.

[16] G. Altarelli and R. Barbieri, Phys. Lett. B253 (1991) 161;

G. Altarelli, R. Barbieri and S. Jadach, Nucl. Phys. B369 (1992) 3; B376 (1992) $444(\mathrm{E})$.

[17] G. Altarelli, R. Barbieri and F. Caravaglios, Nucl. Phys. B405 (1993) 3.

[18] C.P. Burgess, S. Godfrey, M. König, D. London and I. Maksymyk, Phys. Lett. B326 (1994) 276.

[19] I. Maksymyk, C.P. Burgess and D. London, Phys. Rev. D50 (1994) 529.

[20] E. Gates and J. Terning, Phys. Rev. Lett. 67 (1991) 1840.

[21] B. Holdom, Phys. Lett. B105 (1985) 301;

T. Appelquist, D. Karabali and L.C.R. Wijewardhana, Phys. Rev. Lett. 57 (1986) 957;

M. Bando, T. Morozumi, H. So and K. Yamawaki, Phys. Rev. Lett. 59 (1987) 389;

T. Akiba and T. Yanagida, Phys. Lett. B169 (1986) 432.

[22] T. Appelquist, T. Takeuchi, M. Einhorn and L.C.R. Wijewardhana, Phys. Lett. B220 (1989) 223;

B. Holdom, Phys. Lett. B226 (1989) 137;

S. King and D. Ross, Phys. Lett. B236 (1990) 327.

[23] CDF Collaboration, F. Abe et al., Phys. Rev. D50 (1994) 2966; Phys. Rev. Lett. 73 (1994) 225.

[24] N. Evans, Phys. Rev. D49 (1994) 4785. 
[25] CDF and D0 Collaborations, as presented by C.-K. Jung at the 27th Intern. Conf. on High Energy Physics, Glasgow, Scotland, 1994, to appear in the Proceedings.

[26] The LEP Collaborations ALEPH, DELPHI, L3 and OPAL and the LEP Electroweak Working Group, plenary talk given by D. Schaile at the same Conference as Ref. [25].

[27] SLD Collaboration, as presented by M.J. Fero, at the same Conference as Ref. [25].

[28] H. Burkhardt, F. Jegerlehner, G. Penso and C. Verzegnassi, Z. Phys. C43 (1989) 497; F. Jegerlehner, in Proceedings of the 1990 TASI Lectures (Boulder, Colorado, 1990), ed. by P. Langacker and M. Cvetič (World Scientific, Singapore, 1991), p. 476.

[29] S. Catani, in Proceedings of the EPS Conference on High Energy Physics (Marseille, France, 1993), ed. by J. Carr and M. Perrotet (Ed. Frontières, Gif-surYvette, 1994).

[30] Review of Particle Properties, Phys. Rev. D50 (1994) Part I.

[31] W.T. Eadie, D. Drijard, F.E. James, M. Roos and B. Sadoulet, Statistical Methods in Experimental Physics (North Holland, Amsterdam and London, 1971).

[32] M.E. Peskin and T. Tacheuchi, Phys. Rev. Lett. 65 (1990) 964;

R.N. Cahn and M. Suzuki, Phys. Rev. D44 (1991) 3641.

[33] M. Golden and L. Randall, Nucl. Phys. B361 (1990) 3;

B. Holdom and J. Terning, Phys. Lett. B247 (1990) 88;

A. Dobado, D. Espriu and M.J. Herrero, Phys. Lett. B255 (1991) 405.

[34] R. Sundrum and S.D.H. Hsu, Nucl. Phys. B391 (1993) 127.

[35] N.J. Evans and D.A. Ross, Nucl. Phys. B417 (1994) 151.

[36] N.J. Evans, F. King and D.A. Ross, Phys. Lett. B303 (1993) 295. 
[37] R.S. Chivukula, B. Selipsky and E.H. Simmons, Phys. Rev. Lett. 69 (1992) 575 .

[38] N. Kitazawa, Phys. Lett. B313 (1993) 395.

[39] Z. Xiao, L. Wan, G. Lu, J. Yang, W. Xuelei, L. Guo and C. Yue, J. Phys. G20 (1994) 901.

[40] T. Appelquist, T. Tacheuchi, M.B. Einhorn and L.C.R. Wijewardhana, Phys. Lett. B232 (1989) 211;

T. Appelquist and G. Triantaphyllou, Phys. Lett. B278 (1992) 345;

S.F. King and S.H. Mannan, Nucl. Phys. B369 (1992) 119.

[41] T. Appelquist and J. Terning, Phys. Lett. B315 (1993) 139.

[42] M.A. Luty and R. Sundrum, Phys. Rev. Lett. 70 (1993) 529.

[43] T. Yoshikawa, H. Takata and T. Morozumi, Hiroshima Univ. preprint HUPD9406.

[44] R.S. Chivukula, E.H. Simmons and J. Terning, Phys. Lett. B331 (1994) 383.

[45] R.S. Chivukula, E. Gates, E.H. Simmons and J. Terning, Phys. Lett. B311 (1993) 157.

[46] N. Evans, Phys. Lett. B331 (1994) 378.

[47] M.B. Einhorn and D. Nash, Nucl. Phys. B371 (1992) 32.

[48] N. Evans, Swansea Univ. preprints SWAT/33 and SWAT/40.

[49] B. Holdom and R. Lewis, Phys. Rev. D50 (1994) 3491.

[50] E. Eichten and K. Lane, Phys. Lett. B327 (1994) 129;

K. Lane, Boston Univ. preprint BUHEP-94-12.

[51] D0 Collaboration, as presented by P. Grannis at the same Conference as Ref. [25].

[52] J. Ellis, G.L. Fogli and E. Lisi, Phys. Lett. B324 (1994) 173. 


\section{Figure Captions}

Fig. 1: Projections of the four-dimensional space $\left(\epsilon_{1}, \epsilon_{2}, \epsilon_{3}, \epsilon_{b}\right)$ on the six planes $\left(\epsilon_{i}, \epsilon_{j}\right)$, showing the Born approximation (indicated by a star), the projections of the $\Delta \chi^{2}=1$ ellipsoid favoured by precision electroweak data (indicated by solid ellipses), and the regions spanned in the SM for $140 \mathrm{GeV}<m_{t}<200 \mathrm{GeV}, 60 \mathrm{GeV}<M_{H}<$ $1000 \mathrm{GeV}$. Intermediate values in the SM "grid" correspond to $m_{t}=160,180 \mathrm{GeV}$ and $M_{H}=140,400 \mathrm{GeV}$. The dotted lines connect different projections involving the same $\epsilon_{i}$. Note that the data lie far from the Born approximation, but are compatible with the SM, with the possible exception of $\epsilon_{b}$.

Fig. 2: Comparison of the Born approximation (stars), projections of the $\Delta \chi^{2}=$ 1, 4 ellipsoid (solid ellipses), the SM (grid) and the predictions of a one-generation TC model with $N_{T C}=2$, a Dirac technineutrino, $M_{U}=M_{D}, 100 \mathrm{GeV}<M_{E}<600$ $\mathrm{GeV}, 50 \mathrm{GeV}<M_{N}<M_{E}$ and $\xi>1 / 2$ (scattered dots). The TC predictions are added to the SM radiative corrections, using the reference values $m_{t}=170 \mathrm{GeV}$ and $M_{H}=M_{Z}$. Note that the TC predictions are further than the SM from the experimental data. The bold arrows labelled TQ and B indicate possible shifts in the TC predictions of definite sign, and the other (thin) arrows labelled B and NC indicate shifts that are less certain, as discussed in the text.

Fig. 3: As for Fig. 2, but with the corresponding predictions of a one-generation TC model with a Majorana technineutrino. The bold arrows labelled TQ and WTCSETC indicate possible shifts of definite sign, and the other (thin) arrows labelled WTC indicate shifts that are less certain, as discussed in the text. The two sets of arrows in Figs. 1, 2 could be interchanged - they are not specific to the technineutrino being Dirac or Majorana.

Fig. 4: Contours of $\sigma \equiv \sqrt{\Delta \chi^{2}}$ for one-generation models with either Dirac technineutrinos (a), (b) or Majorana technineutrino (c), (d). Note that $\sigma \gtrsim 4.5$ in all of the TC parameter space, to be compared with $\sigma=2.6$ in the SM at the reference point $\left(m_{t}=170 \mathrm{GeV}, M_{H}=M_{Z}\right)$. In the case of techniquark mass degeneracy $\left(M_{U}=M_{D}\right)$, the Dirac and Majorana models fits are comparable; in the case $M_{U}>M_{D}$, however, the Dirac model becomes highly disfavoured. In all cases $\xi=1 / 2$ is assumed. See the text for details. 

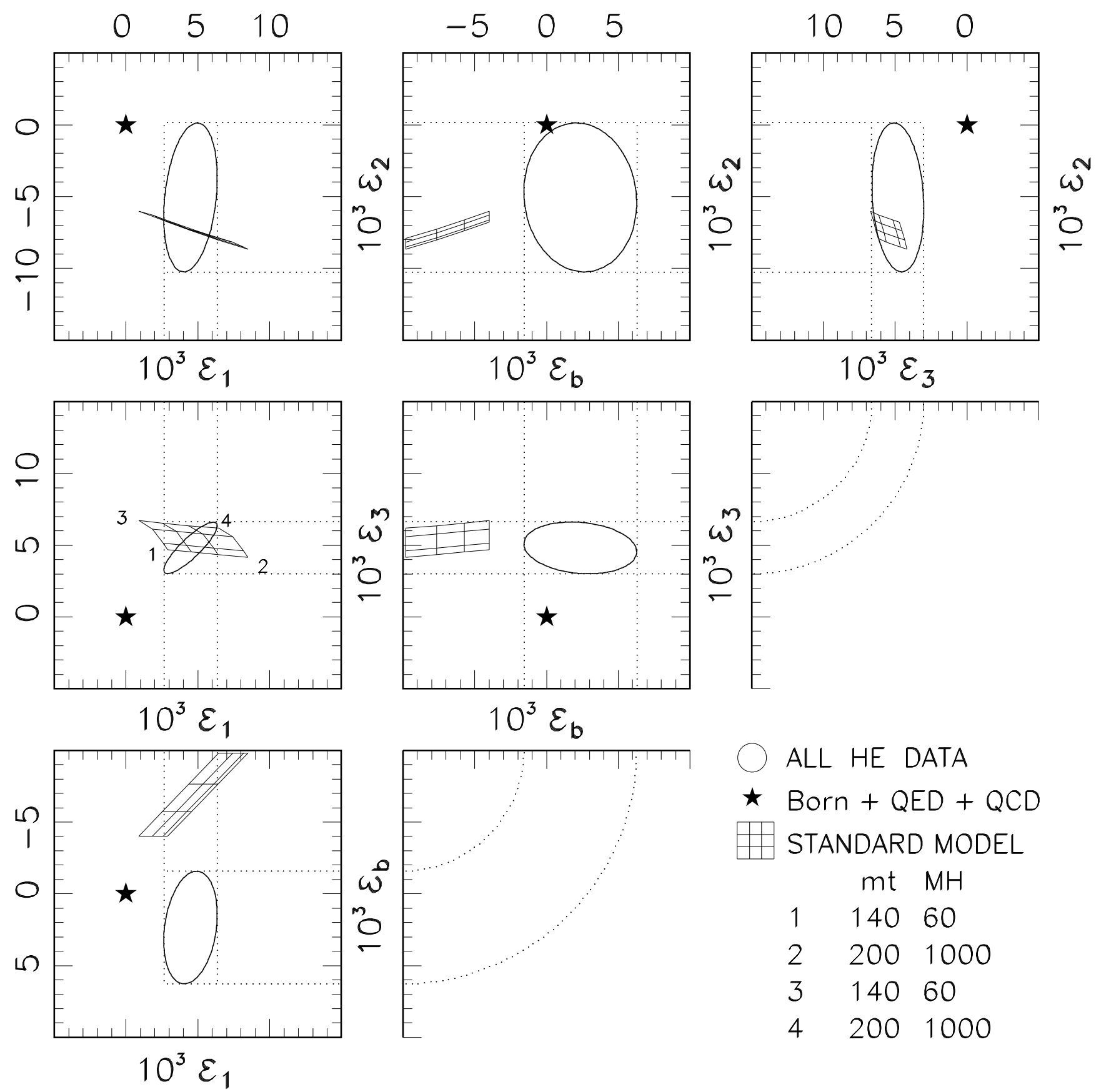

ALL HE DATA

$\star$ Born + QED + QCD

$\#$ STANDARD MODEL

$\begin{array}{rrl} & m t & M H \\ 1 & 140 & 60 \\ 2 & 200 & 1000 \\ 3 & 140 & 60 \\ 4 & 200 & 1000\end{array}$

Fig. 1 


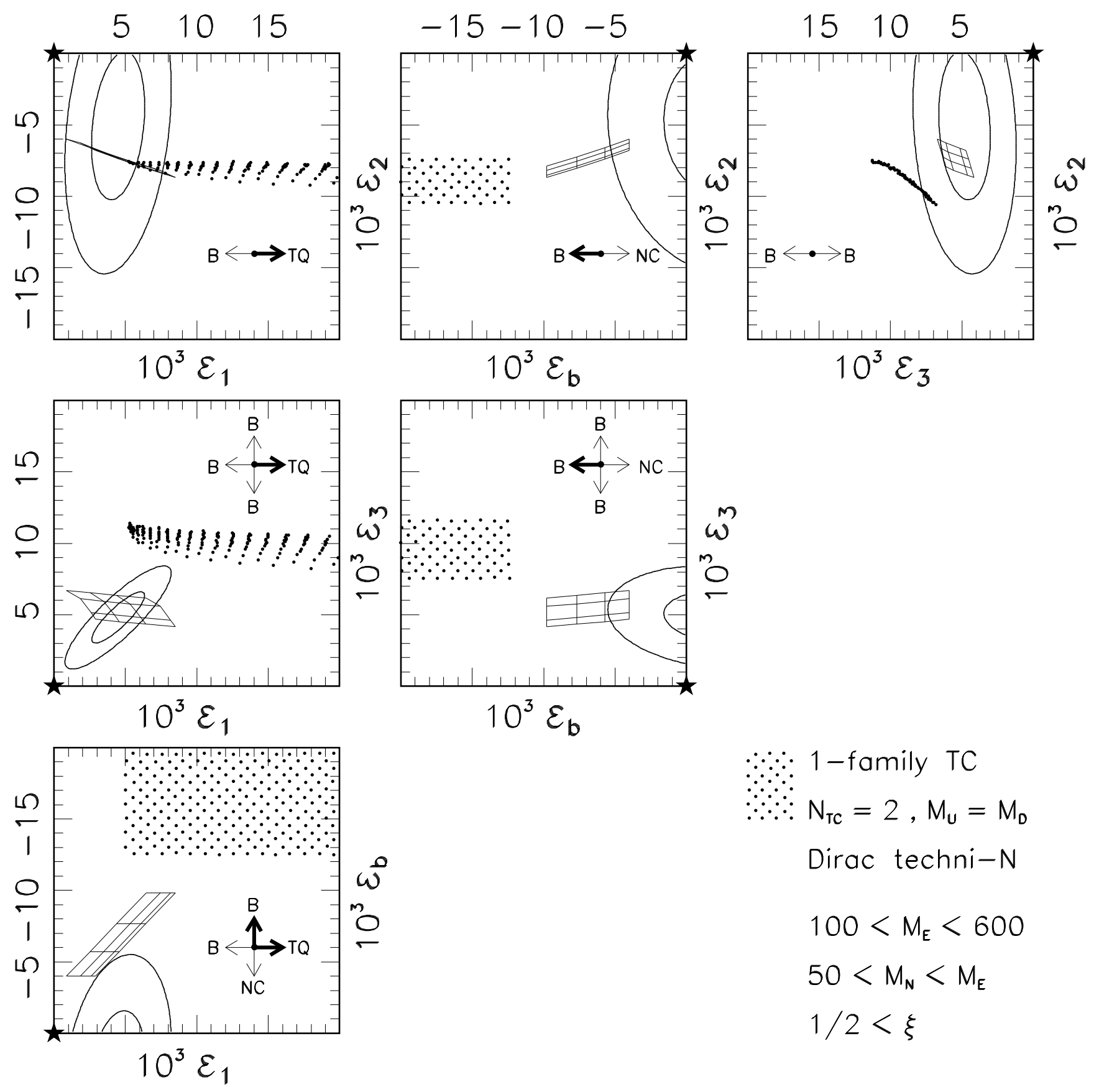

Fig. 2 

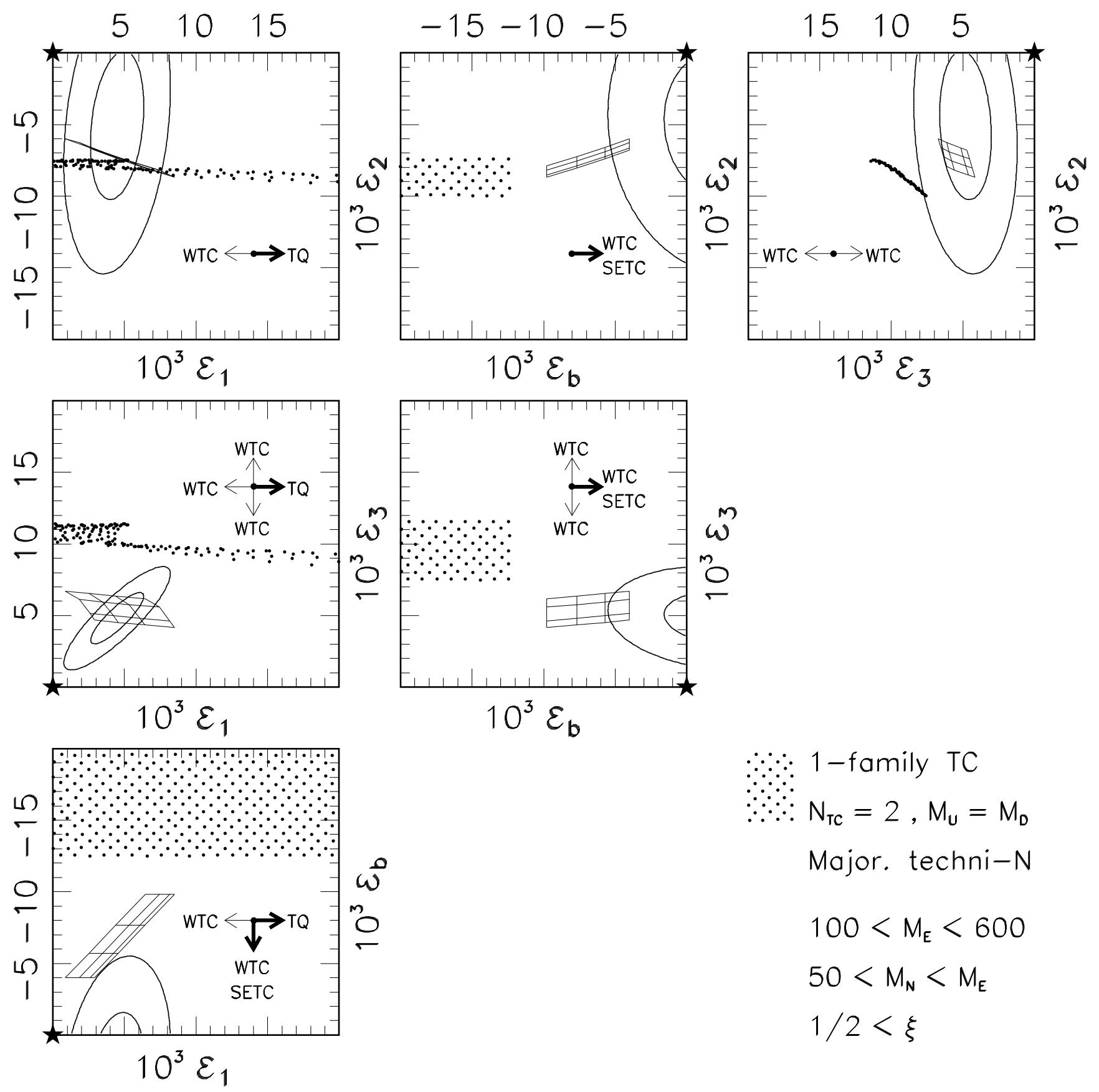

$\because 1-$ family TC

$\because \because \mathrm{N}_{\mathrm{TC}}=2, \mathrm{M}_{\mathrm{U}}=\mathrm{M}_{\mathrm{D}}$

Major. techni-N

$100<M_{E}<600$

$50<M_{N}<M_{E}$

$1 / 2<\xi$

Fig. 3 

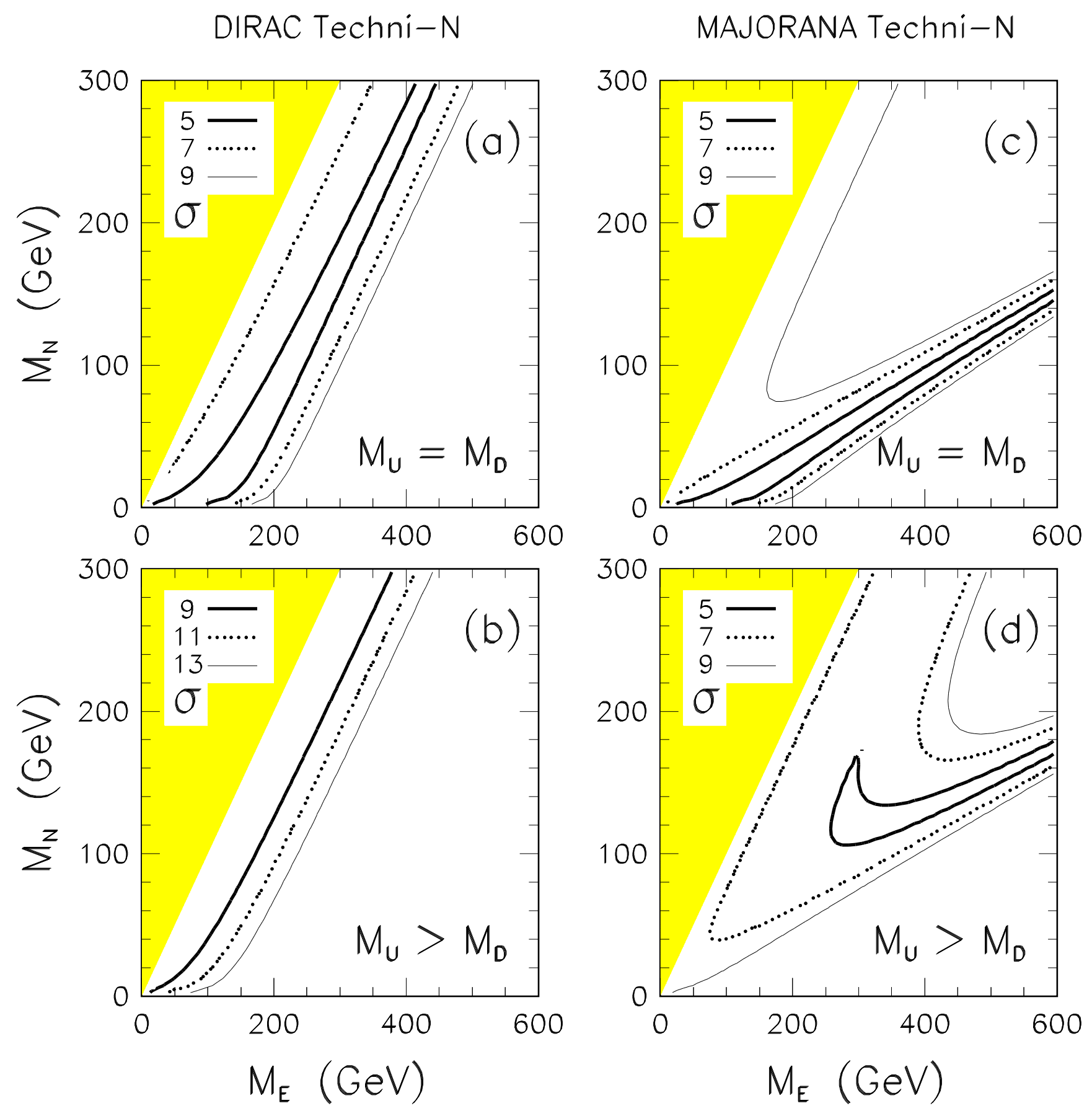

Fig. 4 